\title{
Indicações para o tratamento da má oclusão de classe II em duas fases: revisão sistematizada
}

\author{
Indications for two-phase treatment of class II malocclusion: \\ systematized review \\ Indicaciones para el tratamiento de las maloclusiones de clase II en dos \\ fases: revisión sistematizada \\ Lucas De Carvalho DEPRÁ ${ }^{1}$ \\ Gustavo Henrique Hiroshi do NASCIMENTO ${ }^{1}$ \\ Rodrigo Lorenzi POLUHA ${ }^{2}$ \\ Laurindo Zanco FURQUIM ${ }^{3}$ \\ ${ }^{l}$ Cirurgião-Dentista. Especialista em Ortodontia. Departamento de Odontologia, Universidade Estadual de Maringá-UEM, \\ 87080-000. Maringá-PR, Brasil \\ ${ }^{2}$ Cirurgião-Dentista. Departamento de Odontologia, Universidade Estadual de Maringá-UEM, 87080-000. Maringá-PR, Brasil \\ ${ }^{3}$ Professor do Departamento de Odontologia, Universidade Estadual de Maringá-UEM 87080-000. Maringá-PR, Brasil
}

\begin{abstract}
Resumo
O tratamento da má oclusão de Classe II em duas fases é eficaz em sua correção, entretanto apresenta um tem tempo médio de tratamento maior, sem resultar em benéficos significantes no resultado final. O presente estudo teve como objetivo revisar a literatura a respeito das indicações do tratamento da Classe II em duas fases, apresentando possíveis benefícios adicionais de um tratamento em duas fases, como redução de estresse psicológico e risco a traumas dentários. Grandes reduções de trespasse horizontal em dentadura mista mostraram-se eficazes na melhora da autoestima e qualidade de vida de pacientes vítimas de bullying, assim como na redução da incidência de traumas dentários, tendo como principais beneficiados pacientes mais sensíveis psicologicamente e aqueles com maior risco a traumas dentários.
\end{abstract}

Descritores: Má Oclusão de Angle Classe II; Bullying; Autoimagem; Traumatismos Dentários.

\section{Abstract}

Two-phase Class II treatment has efficacy to correct this malocclusion, however, the main time of treatment is higher in early treatment and also not delivering significant benefits considering the final results. The objective of this study was address physical and psychological aspects of Class II malocclusion individual's development, showing beneffits that can be achieved with early treatment, as reduction of phychological stress and incidency of traumatic dental injuries (TDI). Significant overjet correction in mixed dentition seem to increase self-esteem and oral heath related quality of life in patients suffering with bullying, and is capable of decrease the incidence of TDI. Therefore, early treatment brings benefits to patients who are psychologically sensitive and those at high risk for TDI.

Descriptors: Malocclusion, Angle Class II; Bullying; Self Concept; Tooth Injuries.

\section{Resumen}

El tratamiento de las maloclusiones de Clase II en dos fases es eficaz en su corrección, sin embargo, presenta uno tiene tiempo promedio de tratamiento mayor, sin resultar en beneficiosos significantes en el resultado final. El presente estudio tuvo como objetivo revisar la literatura acerca de las indicaciones del tratamiento de la Clase II en dos fases, presentando posibles beneficios adicionales de un tratamiento en dos fases, como reducción de estrés psicológico y riesgo a traumas dentales. Las grandes reducciones de traspaso horizontal en dentadura mixta se mostraron eficaces en la mejora de la autoestima y calidad de vida de pacientes víctimas de bullying, así como en la reducción de la incidencia de traumas dentales, teniendo como principales beneficiadas pacientes más sensibles psicológicamente y aquellos con mayor riesgo traumas dentales.

Descriptores: Maloclusión de Angle Clase II; Acoso Escolar; Autoimagen; Traumatismos de los Dientes.

\section{INTRODUÇÃO}

A má oclusão de Classe II pode ser diagnosticada precocemente, entretanto a variedade de padrões de crescimento e a individualidade de cada paciente ${ }^{1-4}$ tornam difíceis a previsibilidade do caso e consequentemente, dificultam decisões quanto ao momento ideal para intervir e o método mais eficiente 5 .

Quando o tratamento é iniciado precocemente, a relação de Classe II dentária é corrigida através de dispositivos funcionais removíveis ou fixos ainda na dentadura mista. Após a primeira fase do tratamento, é necessário o uso de aparelhos fixos em dentadura permanente, para alinhamento, nivelamento e finalização do caso. O tratamento em duas fases acaba sendo mais prolongado em média, do que aqueles realizados em uma fase, utilizando-se dispositivos fixos ${ }^{6,7}$, exigindo maior esforço para manter a motivação do paciente e seus familiares. Apesar de apresentar mudanças esqueléticas significativas ao término de sua primeira fase, os benefícios não são evidentes ao final da segunda fase de tratamento ${ }^{8-10}$. Dolce et al. ${ }^{7}$ observaram que as mudanças no crescimento ósseo decorrente do tratamento precoce da Classe II foram de baixa magnitude. Tulloch e colaboradores (1998) obtiveram melhor relação oclusal ao final do tratamento em uma fase, quando comparado ao tratamento em duas fases ${ }^{11}$.

Com maior tempo médio de tratamento sem obtenção de melhor relação entre as bases ósseas e oclusão dentária ${ }^{11}, 0$ tratamento precoce não deveria ser visto como a maneira mais eficiente de tratar a Classe II. No entanto, considerando aspectos individuais de cada criança ${ }^{12}$, como características físicas e psicológicas de seu desenvolvimento, a intervenção em duas fases ainda tem aplicabilidade. A maior parte da literatura em ortodontia concentra-se nos métodos de tratamentos empregados e seus aspectos biológicos, deixando de lado aspectos físicos e psicológicos ${ }^{13}$ que podem, direta ou indiretamente, trazer impacto social ao paciente, afetando sua qualidade de vida. O surgimento de tratamentos mais eficientes levam os profissionais a definirem planos de tratamento desconsiderando as expectativas dos pacientes relacionadas às suas interações sociais. Uma visão mais ampla e detalhada do paciente permite ao ortodontista a indicação do tratamento precoce de anomalias que, por aparência ou funcionalidade, tornem-se um obstáculo ao bem estar físico e psicológico do paciente ${ }^{14}$. Portanto, o presente trabalho objetiva revisar a literatura a respeito das indicações para o tratamento em duas fases da Classe II.

\section{MATERIAL E MÉTODO}

Foram realizadas buscas nas bases de dados Pubmed, Lilacs, Scielo, Science Direct, utilizando-se os seguintes descritores em língua inglesa e portuguesa: "Má Oclusão de Angle Classe II", "Avanço Mandibular", "Autoimagem", "Autoestima", "Qualidade de Vida", "Traumatismos 
Dentários", "Fraturas dos Dentes". Foram incluídos artigos de revisão de literatura, revisão sistemática, metanálise, estudos clínicos randomizados e demais trabalhos pertinentes ao estudo, publicados no período de janeiro de 1959 a janeiro de 2018.

\section{REVISÃO DA LITERATURA e DISCUSSÃO}

\section{- Fatores Psicológicos}

Entre as anomalias físicas, aquelas localizadas na face são as que causam maior impacto na autoavaliação do paciente ${ }^{15}$. Devido a grande visibilidade, mesmo pequenas alterações nesta região podem causar estresse psicológico e ansiedade $^{15}$. Isso ocorre porque a aparência facial é uma importante variável na formação das primeiras impressões ${ }^{16}$, podendo gerar preconceitos quanto a personalidade ou capacidade intelectual do indivíduo de acordo com suas características físicas ${ }^{17}$.Pessoas tidas como menos atraentes são percebidas como menos populares, pouco amigáveis e sociáveis ${ }^{18}$. Estes fatores geram uma insatisfação pessoal com a aparência, abaixando sua autoestima e impactando sua vida social e consequentemente diminuindo sua qualidade de vida $^{15}$.

A sociedade contemporânea segue conceitos socioculturais estipulados, que ditam padrões de comportamento, consumo e beleza. A crescente exigência estética expõe desde a infância o indivíduo ao julgamento de seus pares. As crianças que, física ou socialmente diferem dos demais, são muitas vezes vítimas de bullying ${ }^{19,20}$. O bullying é uma forma de comportamento agressivo e caracteriza-se pela exposição frequente de uma criança a ações negativas de um ou mais colegas ${ }^{21}$. Tais ações podem ser diretas, como agressão física, apelidos e xingamentos, ou indiretas como fofocas, rumores ou até exclusão do círculo social ${ }^{22}$.

O sorriso tem grande participação na aparência geral da face. Devido a sua influência na formação das primeiras impressões, anomalias dentárias geram preconceitos e são relacionadas a pessoas com baixa atratividade social ${ }^{20}$, além de serem relatadas como a quarta maior causa de bullying entre jovens, abaixo de altura, peso e aparência do cabelo ${ }^{22}$. O julgamento dos colegas, que culmina em provocações, gera uma insatisfação estética à criança e abaixa sua autoestima ${ }^{23}$. Já indivíduos mais sensíveis, têm sua qualidade de vida reduzida, vida social e emocional afetada e podem carregar danos psicológicos até a vida adulta ${ }^{24-26}$.

As más oclusões mais relacionadas ao bullying e insatisfação estética são trespasse horizontal acentuado, mordida profunda, apinhamento dentário ${ }^{27}$ e protrusão dos incisivos, características que podem ser observadas na Classe II. Quando a discrepância anteroposterior é grande, as características oclusais estão acompanhadas de um padrão facial mais convexo. Linha queixo-pescoço encurtada, eversão do lábio inferior, sulco mentolabial mais evidente, ausência de selamento labial passivo e a protrusão dos incisivos superiores são aspectos faciais que podem estar presentes no indivíduo, que muitas vezes se apresenta relutante em sorrir, devido a sua aparência e todo o impacto social acarretado ${ }^{28}$.

Quando comparada ao tratamento em uma fase, o tratamento precoce da Classe II mostra-se menos eficiente, pois é mais prolongado e não apresenta resultados superiores. Entretanto, este acontece com um indivíduo em desenvolvimento e por isso, o impacto psicológico causado pela má oclusão e os benefícios do tratamento ortodôntico devem ser considerados. É desejável que os danos causados por uma má oclusão severa no desenvolvimento de uma criança sejam reduzidos ou prevenidos ${ }^{16}$. Nas últimas décadas, vários estudos ${ }^{9,11,13}$ relacionaram o tratamento ortodôntico a autoestima e ao bem-estar social. Essa relação é complexa porque o bem-estar social e psicológico de um indivíduo é multifatorial, tornando os resultados controversos e os benefícios do tratamento ortodôntico incertos.

Em estudo longitudinal, com acompanhamento de 20 anos, Shaw et al. $^{13}$, revelaram que não houve aumento significante da autoestima em pacientes que receberam tratamento ortodôntico, quando comparados àqueles que não foram tratados ${ }^{13}$. Uma explicação para este resultado, é que o desenvolvimento do jovem, tanto fisiológico quanto psicológico, o torna mais tolerante a provocações e mais capacitado a lidar com sua aparência, sem que esta interfira na sua qualidade de vida ${ }^{17}$. Dann et al. ${ }^{29}$ não obtiveram mudanças na autoestima de crianças com o uso de aparelhos funcionais no tratamento da Classe $\mathrm{II}^{29}$. Este resultado contradiz estudo mais recente, onde O'Brien et al. ${ }^{26}$ perceberam melhoras no autoconceito dos pacientes após término da primeira fase do tratamento ${ }^{26}$. Isso ocorre, porque no primeiro, ocorreu uma diminuição média de $2.1 \mathrm{~mm}$ no trespasse horizontal, enquanto no segundo, a redução média foi de $6.6 \mathrm{~mm}$. Acredita-se que a alta incidência de bullying em pacientes com Classe II severa seja motivada pelas alterações faciais causadas pela grande discrepância anteroposterior. Logo, uma correção mais eficiente do trespasse horizontal traria maiores benefícios. Agou et al. ${ }^{30}$ também obtiveram uma melhora no bem-estar social de jovens entre 11 e 14 anos após intervenção ortodôntica. De Oliveira e Sheiham ${ }^{31}$ observaram em população brasileira, melhora na qualidade de vida relacionada à saúde bucal em adolescentes de 15 e 16 anos que concluíram o tratamento ortodôntico, quando comparados àqueles que não passaram por tratamento ortodôntico ou ainda não o concluíram. Concomitante a isso, cada vez mais os aparelhos dentários são aceitos entre os jovens, sendo considerada parte de uma aparência bucal normal entre adolescentes de 11 a 15 anos de idade $^{32}$. Esta mudança minimiza a preocupação de crianças e pais quando aos dispositivos ortodônticos tornarem-se a causa do bullying ao invés de trazer benefícios nesta questão.

Adolescentes vítimas de bullying devido a aparência dentária relatam um impacto negativo na autoestima e na qualidade de vida relacionada à saúde bucal. Características que causem este comportamento agressivo, como trespasse horizontal acentuado, podem ser interpretadas como uma desvantagem social, o que torna compreensível o desejo da criança e seus familiares pelo tratamento, visto que uma correção mesmo que parcial, pode trazer benefícios psicológicos significantes a uma criança sensível ${ }^{16}$.

A maior influência para que os pacientes busquem tratamento ortodôntico é a indicação de outro cirurgiãodentista $^{16}$. Por isso, é fundamental que odontopediatras e ortodontistas estejam atentos aos sintomas relacionados a estresse psicológico, assim como doenças psiquiátricas em pacientes com má oclusão severa ${ }^{15^{5}}$. Nestes casos, a intervenção ortodôntica precoce tem indicação e pode trazer benefícios reais para o bem-estar social do paciente.

- Trauma Dentário

O trauma dentário é uma ocorrência muito estudada e debatida no meio odontológico, em virtude da sua grande prevalência na população mundial e dos prejuízos sociais e econômicos acarretados por estes acidentes. Muitos estudos chegaram a números impactantes, apontando que a incidência de trauma na dentadura decídua é de aproximadamente $30 \%$, enquanto na dentadura permanente atinge cerca de $20 \%$ das crianças e adolescentes ${ }^{33,34}$. Entre os fatores que podem influenciar na prevalência do trauma dentário, estão idade, sexo, atividades de risco, comportamento e características socioeconômicas. Durante cada fase do desenvolvimento humano, são praticadas diferentes atividades e apresentados comportamentos que podem expor o indivíduo a riscos. Devido ao equilíbrio e coordenação motora limitada durante os primeiros anos de vida, a criança sofre grande quantidade de quedas, tornando esta a principal causa de trauma dentário entre pré-escolares. Em crianças com idade escolar as lesões 
costumam ser provenientes da prática esportiva. Já em adolescentes e adultos jovens, agressões e acidentes automobilísticos figuram como principais fatores de risco ${ }^{35}$. A prática de esportes e outras atividades de risco, associada ao comportamento agitado típico da idade, faz com que haja um pico de risco ao trauma dentário em crianças do sexo masculino ${ }^{36}$ entre 8 e 11 anos $^{37-40}$.

Além destas variáveis, muitos autores listam algumas características oclusais e faciais como determinantes para o aumento do risco a fratura dos dentes anteriores como: protrusão dos incisivos superiores ${ }^{41,42}$, trespasse horizontal acentuado $^{44}$ e ausência de selamento labial ${ }^{42-45}$. Tais características estão comumente presentes em pacientes com má oclusão de Classe II, divisão 1, também associada a pacientes com dentes traumatizados. Apesar de inúmeros estudos que correlacionam a Classe II ao trauma dentário, os resultados são contraditórios e muitas vezes inconclusivos. Koroluk et al. $^{46}$ não encontraram relação entre trespasse horizontal acentuado e lesões traumáticas, coincidindo com os achados de Stokes et al. $^{47}$. Ao tratarem pacientes Classe II, Chen et al. ${ }^{48}$ não obtiveram alteração do risco a traumas em seus pacientes.

Por outro lado, muitos são os estudos nos quais pacientes com grandes trespasses horizontais apresentaram significativamente mais episódios de trauma dentário. Lewis ${ }^{49}$ observou que incisivos superiores traumatizados se encontravam mais protruídos no arco, característica esta que viria a ser confirmada por outros autores nas décadas seguintes, que apontaram risco aumentado em crianças com trespasses horizontais acima de $6 \mathrm{~mm}^{42,50}$. Petti ${ }^{51}$ realizou uma metanálise e concluiu que a presença de trespasse horizontal acentuado pode dobrar ou até triplicar os riscos ao trauma dentário. Esta associação ocorre devido as características do paciente portador da má oclusão de Classe II, divisão 1 . Devido a grande discrepância anteroposterior, existe trespasse horizontal acentuado e protrusão dos incisivos superiores. Além da posição avançada do dente, muitas vezes o paciente possui lábios hipotônicos e ausência de selamento labial, aumentando assim sua exposição e tornando-o mais susceptível a fraturas e danos.

Considerando a Classe II fator significativo de aumento de risco ao trauma dentário ${ }^{52,53}$, seu tratamento precoce se torna uma estratégia para diminuição e prevenção de $\operatorname{riscos}^{53}$. Seus benefícios foram observados em revisão sistemática que apontou uma diminuição na incidência de novos traumas incisais em pacientes submetidos ao tratamento de Classe II e consequente diminuição de trespasse horizontal $^{54}$.

Assumindo os benefícios potenciais da intervenção precoce em Classe II, sua eficácia depende da época do início do tratamento ${ }^{48}$. A fase que apresenta maior risco a trauma, compreende o período intertransitório e parte do segundo período transitório. Por isso, para uma redução do trespasse horizontal e prevenção de acidentes envolvendo os dentes anteriores, é necessário que o tratamento seja iniciado logo após a irrupção dos incisivos superiores ${ }^{46}$.

Chen et al. ${ }^{48}$ observaram que pacientes com histórico de trauma têm maior chance de reincidência. Indivíduos com traumas múltiplos costumeiramente relatam episódios traumáticos mais cedo que os demais, com 6 anos em média ${ }^{55}$ além de apresentarem características comportamentais relacionadas a estresse psicológico, dificuldade de aprendizado, doenças e limitações físicas ${ }^{48}$. Apesar da maioria dos traumas resultarem apenas em danos a esmalte e, em menor número dentina, e apresentam bom prognóstico ${ }^{48,56,57}$, traumas menores podem causar complicações consideráveis em casos de reincidência ou tratamento inadequado ${ }^{56,57}$.

Tais características comportamentais e psicológicas do paciente podem ser observadas durante a consulta odontológica e seu histórico coletado em exame clínico detalhado, tornando possível a identificação de pacientes com alto risco ao trauma dentário e a indicação de tratamento adequado objetivando a redução dos mesmos.

\section{CONCLUSÃO}

O tratamento da Classe II em duas fases pode ser considerado menos eficiente do que o tratamento em uma fase, entretanto o tratamento precoce ainda tem suas indicações. Pacientes mais sensíveis podem levar os impactos psicológicos decorrentes do bullying até a vida adulta. Correções de trespasse horizontal que impactem positivamente a estética facial podem trazer benefícios significativos na autoestima, bem-estar social e qualidade de vida destes pacientes.

Indivíduos que praticam atividades de risco apresentam histórico de trauma dentário ou têm características físicas e psicológicas que corroborem para tal quadro, apresentam risco aumentado para trauma dos dentes anteriores. Quando associados a trespasse horizontal acentuado e protrusão incisiva, o tratamento precoce é indicado, sendo capaz de evitar novos episódios traumáticos.

Cabe ao ortodontista enxergar o paciente além de sua má oclusão. Exame clínico minucioso é capaz de identificar características físicas e psicológicas que leve ao plano de tratamento ideal, o que trará melhor custo benefício para o paciente.

\section{REFERÊNCIAS}

1. Almeida MR, Pereira ALP, Almeida RR, Almeida-Pedrin RR, Silva Filho Og. Prevalência de má oclusão em crianças de 7 a 12 anos de idade. Dental Press J Orthod. 2011; 16(4):123-31.

2. Bjork A. Prediction of mandibular growth rotation. Am J Orthodontics. 1969; 55(6):585-99.

3. Carter NE. Dentofacial changes in untreated Class II division 1 subjects. B J Orthod. 1987; 14(4):225-34.

4. Mills JRE. The effect of functional appliances on the skeletal pattern. B J Orthod. 1991; 18(4):267-75.

5. Proffit WR, Tulloch JFC. Preadolescent Class II problems:Treat now or wait? Am J Orthod Dentofacial Orthop. 2002; 121(6): 560-62.

6. Livieratos FA, Johnson LE. A comparison of one-stage and two-stage nonextraction alternatives in matched Class II samples. Am J Orthod Dentofacial Orthop. 1995; 108(2):118-31.

7. Dolce C, McGorray SP, Brauzeau L, King GJ, Wheeler TT. Timing of Class II treatment: Skeletal changes comparing 1-phase and 2-phase treatment. Am J Orthod Dentofacial Orthop. 2007; 132(4):481-89.

8. Tulloch JF, Phillips C, Koch G, Proffit WR. The effect of early intervention on skeletal pattern in Class II malocclusion: a randomized clinical trial. Am J Orthod Dentofacial Orthop. 1997; 111(4):391-400.

9. Tulloch JF, Proffit WR, Phillips C. Influences on the outcome of early treatment for Class II malocclusion. Am J Orthod Dentofacial Orthop. 1997; 111(5):533-42.

10. von Bremen J, Pancherz H. Efficiency of early and late Class II Division 1 treatment. Am J Orthod Dentofacial Orthop. 2002; 121(1):31-7.

11. Tulloch JF, Phillips C, Proffit WR. Benefit of early Class II treatment: progress report of a two-phase randomized clinical trial. Am J Orthod Dentofacial Orthop. 1998; 113(1):62-72.

12. Tulloch JFC, Proffit WR, Phillips C. Outcomes in a 2phase randomized clinical trial of early Class II treatment. Am J Orthod Dentofacial Orthop. 2004; 125(6):657-67.

13. Shaw WC, Richmond S, Kenealy PM, Kingdon A, Worthington H. A 20-year cohort study of health gain from orthodontic treatment. Psychological outcome. Am J Orthod Dentofacial Orthop. 2007; 132(2):146-57. 
14. World Health Organization. Standardisation of reporting of dental diseases and conditions. Report of an expert committee on dental health. Geneva: WHO; 1962.

15. Jenny J, Cons NC, Kohout FJ, Frazier PJ. Test of a method to determine socially acceptable occlusal conditions. Comm DentOral Epidemiol. 1980; 8(8):424-33.

16. Shaw WC. The influence of children's dentofacial appearance on their social attractiveness as judged by peers and lay adults. Am J Orthod. 1981; 79(4):399-415.

17. Zhang $M$, Mcgrath $C$, Hägg $U$. The impact of malocclusion and its treatment on quality of life: a literature review. Int J Paediatr Dent. 2006; 16(6):381-7.

18. Shaw WC, Meek SC, Jones DS. Nicknames, teasing, harrassment and de salience of dental features among schoolchildren. Brit J Orthod. 1980; 7(2):75-80.

19. Phillips C, Bennett ME, Broder HL. Dentofacial disharmony: Psychological status of patients seeking treatment consultation. The Angle Orthodontist. 1998; 68(6):547-55.

20. Kilpeläunen PV, Phillips C, Tulloch JF. Anterior tooth position and motivation for early treatment. Angle Orthod. 1993; 63(3):171-74.

21. Olweus D. Bullying at school: basic facts and effects of a school based intervention program. J Child Psychol Psychiatry. 1994; 35(7):1171-90.

22. van der Wal MF, de Wit CA, Hirasing RA. Psychosocial health among young victims and offenders of direct and indirect bullying. Pediatrics. 2003; 111(6 Pt 1): 1312-17.

23. Evans R, Shaw W. Preliminary evaluation of an illustrated scale for rating dental attractiveness. Eur J Orthod. 1987; 9(4):314-18.

24. O'Brien K, Wright J, Conboy F, Chadwick S, Connolly I, Cook P et al. Effectiveness of early orthodontic treatment with the Twin-block appliance: a multicenter, randomized, controlled trial. Part 2: psychosocial effects. Am J Orthod Dentofacial Orthop. 2003; 124(5):488-94.

25. Foster Page LA, Thomson WM, Jokovic A, Locker D. Validation of the Child Perceptions Questionnaire (CPQ 11-14). J Dent Res. 2005; 84(7):649-52.

26. O'Brien C, Benson PE, Marshman Z. Evaluation of a quality of life measure for children with malocclusion. Journal of Orthodontics. 2007; 34(3):185-93.

27. Gosney MB. An investigation into some of the factors influencing the desire for orthodontic treatment. $\mathrm{Br} \mathrm{J}$ Orthod. 1986; 13(2):87-94.

28. Seehra J, Newton JT, Dibiase AT. Interceptive orthodontic treatment in bullied adolescents and its impacto $n$ self-esteem and oral-health-related quality of life. Eur J Orthod. 2013; 35(5):615-21.

29. Dann C 4th, Phillips C, Broder HL, Tulloch JF. Selfconcept, Class II malocclusion, and early treatment. Angle Orthod. 1995; 65(6):411-16.

30. Agou S, Locker D, Muirhead V, Tompson B, Streiner DL. Does psychological well-being influence oral-healthrelated quality of life reports in children receiving orthodontic treatment? Am J Orthod Dentofacial Orthop. 2011; 139(3):369-77.

31. De Oliveira CM, Sheiham A. Orthodontic treatment and its impact on oral health-related quality of life in Brazilian adolescents. J Orthod. 2004; 31(1):20-7.

32. Patel A, Rodd HD, Baker SR, Marshman Z, Robinson PG, Benson PE. Are social judgements made by children in relation to orthodontic appliances? J Orthod. 2010; 37(2):93-9.

33. Andreasen JQ, Ravn JJ. Epidemiology of traumatic dental injuries to primary and permanent teeth in a Danish population sample. Int J Oral Surg. 1972; 1(5):235-39.

34. Granville-Garcia AF, Vieira IT, Siqueira MJ, de Menezes VA, Cavalcanti AL. Traumatic dental injuries and associated factors among Brazilian preschool children aged 1-5 years. Acta Odontol Latinoam. 2010; 23(1):47-52.

35. Glendor U. Aetiology and risk factors related to traumatic dental injuries - a review of the literature. Dent Traumatol. 2009; 25(1): 19-31.

36. Traebert J, Peres MA; Blank V, Böell Rda S, Pietruza JA. Prevalence of traumatic dental injury and associated factors among 12-year-old school children in Florianopolis, Brazil. Dent Traumatol. 2003; 19(1):15-8.

37. Bauss O, Rohling J, Schwestka-Polly R. Prevalence of traumatic injuries to the permanent incisors in candidates for orthodontic treatment. Dent Traumatol. 2004; 20(2):61-6.

38. Skaare AB, Jacobsen I. Dental injuries in Norwegians aged 7-18 years. Dent Traumatol. 2003; 19(2):67-71.

39. Oikarinen K, Kassila O. Causes and types of traumatic tooth injuries treated in a public dental health clinic. Endod Dent Traumatol. 1987; 3(4):172-77.

40. Grimm S, Frazão P, Antunes JL, Castellanos RA, Narvai PC. Dental injury among Brazilian schoolchildren in the state of São Paulo. Dent Traumatol. 2004; 20(3):134-38.

41. Eichenbaum IW. A correlation of traumatized anterior teeth occlusion. J Dent Child. 1963; 30:229-36.

42. O'Mullane DM. Some factors predisposing to injuries of permanent incisors in school children. Br Dent J. 1973; 134(8):328-32.

43. Otuyemi OD. Traumatic anterior dental injuries related to incisor overjet and lip competence in 12-year-old Nigerian children. Int J Paediatr Dent. 1994; 4(2):81-5.

44. Brin I, Ben-Bassat Y, Heling I, Brezniak N. Profile of an orthodontic patient at risk of dental trauma. Endod Dent Traumatol. 2000; 16(3):111-15.

45. Burden DJ. An investigation of the association between overjet size, lip coverage, and traumatic injury to maxillary incisors. Eur J Orthod. 1995; 17(6):513-17.

46. Koroluk LD, Tulloch JFC, Phillips C. Incisor trauma and early treatment for Class II Division 1 malocclusion. Am J Orthod Dentofacial Orthop. 2003; 123(2):117-25.

47. Stokes AN, Loh T, Teo CS, Bagramian RA. Relation between incisal overjet and traumatic injury: a case control study. Endod Dent Traumatol. 1995; 11(1):2-5.

48. Chen DR, McGorray SP, Dolce C, Weeler TT. Effect of early Class II treatment on the incidence of incisor trauma. Am J Orthod Dentofacial Orthop. 2011; 140(4):155-60.

49. Lewis TE. Incidence of fractured anterior teeth as related to their protrusion. Angle Orthod. 1959; 29(2):128-31.

50. Ghose LJ, Baghdady VS, Enke H. Relation of traumatized permanent anterior teeth to occlusion and lip condition. Community Dent Oral Epidemiol. 1980; 8(7):381-84.

51. Petti S. Over two hundred million injuries to anterior teeth attributable to large overjet: a meta-analysis. Dent Traumatol. 2015; 31(1):1-8.

52. Bauss O, Freitag S, Röhling J, Rahman A. Influence of overjet and lip coverage on the prevalence and severity of incisor trauma. J Orofac Orthop. 2008; 69(6):402-10.

53. Artun J, Behbehani F, Al-Jame B, Kerosuo H. Incisor trauma in an adolescent Arab population. Prevalence, severity and occlusal risk factors. Am J Orthod Dentofacial Orthop. 2005; 128(3):347-52.

54. Tiruvenkatachari B, Harrison J, Worthington H, O'Brien K. Early orthodontic treatment for Class II malocclusion reduces the chance of incisal trauma: Results of a Cochrane systematic review. Am J Orthod Dentofacial Orthop. 2015; 148(1):47-59.

55. Pissiotis A, Vanderas AP, Papagiannoulis L. Longitudinal study on types of injury, complications and treatment in permanent traumatized teeth with single and 
multiple dental trauma episodes. Dent Traumatol. 2007 23(4):222-5.

56. Zadik D, Chosack A, Eidelman E. The prognosis of traumatized permanent anterior teeth with fracture of enamel and dentin. Oral Surg Oral Med Oral Pathol. 1979; 47(2):173-75.

57. Hedegard B, Stalhane I. A study of traumatized permanent teeth in children aged 7-15 years. Sven Tandlak Tidskr. 1973; 66(5):431-50.

\section{CONFLITO DE INTERESSES}

Os autores declaram não haver conflitos de interesse.

\section{AUTOR PARA CORRESPONDÊNCIA}

Rodrigo Lorenzo Poluha

rodrigopoluha@gmail.com 